\title{
Histological transformation from gastric mucosa-associated lymphoid tissue lymphoma to gastric diffuse large B-cell lymphoma
}

\author{
XIAOWU LI $^{1 *}$, QINGLIANG MENG ${ }^{2 *}$, SANYUAN SUN $^{1}$, ZHONGLI ZHAN $^{3}$, \\ LIANYU ZHANG ${ }^{3}$, BAOCHUN SUN ${ }^{3}$ and YIZHUO ZHANG ${ }^{4}$ \\ Departments of ${ }^{1}$ Medical Oncology and ${ }^{2}$ Oncological Surgery, Xuzhou Central Hospital, \\ Xuzhou, Jiangsu 277100; Departments of ${ }^{3}$ Pathology and ${ }^{4}$ Hematology, Tianjin Medical University \\ Cancer Institute and Hospital, Tianjin 300060, P.R. China
}

Received October 17, 2016; Accepted April 13, 2017

DOI: $10.3892 / \mathrm{ol} .2017 .6402$

\begin{abstract}
This study evaluated the clinical features, treatment and prognosis in Chinese patients with histological transformation (HT) from gastric mucosa-associated lymphoid tissue lymphoma to gastric diffuse large B-cell lymphoma. We reviewed the medical records of 71 patients diagnosed with HT between 2001 and 2013, retrospectively. Patients had a median age of 56 years. The ratio of sex (male:female) was 1.3:1. The clinical course was often insidious, lacking specific clinical presentation. Macroscopically, the antrum was the most commonly involved site. Thirty-one patients (45\%) presented at stage I, and $25(35 \%)$ presented with local $(18 / 71,25 \%)$ or distant $(7 / 71,10 \%)$ nodal involvement. There were also stage IIE $(9 / 71,12 \%)$ and stage IV $(6 / 71$, $8 \%)$ patients with advanced stages. For all 71 patients, the 5 -year progression-free survival (PFS) and overall survival (OS) estimates were 50 and 56\%, respectively. There was no statistical difference in 5-year PFS and OS estimates between patients receiving Helicobacter pylori (H.pylori) containing eradication (HPE) $(\mathrm{p}=0.189)$ and those receiving non-HPE $(\mathrm{p}=0.359)$. Upon the Cox regression model, advanced stages were the only independent prognostic factors associated with shorter PFS, and m-IPI was independently associated with shorter PFS and OS. There was no specific clinical manifestation for patients with HT. HPE is thus a promising therapeutic approach for such patients. Moreover, advanced stages and m-IPI significantly influenced patient outcome.
\end{abstract}

Correspondence to: Dr Sanyuan Sun, Department of Medical Oncology, Xuzhou Central Hospital, 199 Jiefang Road, Quan Shan, Xuzhou, Jiangsu 277100, P.R. China

E-mail: sunsanyuan1017@163.com

${ }^{*}$ Contributed equally

Key words: histological transformation, large B-cell lymphoma

\section{Introduction}

Primary gastric lymphoma is the most common form of extranodal lymphoma, including gastric mucosa-associated lymphoid tissue (gMALT) lymphoma and gastric diffuse large B-cell lymphoma (gDLBCL) (1). Histological transformation (HT) from gMALT lymphoma to gDLBCL has recently been reported (2-4), and molecular mechanisms for transition exists. Kaneko et al suggested the model for gMALT lymphoma development and progression (4). The pathogenesis of gDLBCL in that study, has two distinct pathways, some gDLBCL may arise from indolent gMALT via the step of H. pylori dependent as HT, and the residual gDLBCLs may develop along antigen-independent pathways as de novo gDLBCL. Some gDLBCL may arise from indolent gMALT via the step of $H$. pylori-dependent HT, and the residual gDLBCLs may develop along antigen-independent pathways as de novo gDLBCL. However, information concerning patients with HT is very limited.

Thus, a retrospective analysis of patients with HT was conducted by investigating their clinical features, treatment and prognosis.

\section{Materials and methods}

Patients and staging procedures. We reviewed 71 patients with HT who were diagnosed at the Tianjin Medical University Cancer Institute and Hospital and the Xuzhou Central Hospital from January 2001 to June 2013. The diagnostic criteria for HT were based on the World Health Organization (WHO) classification system for hematologic malignancies (1) that manifest as lymphomatous foci containing DLBCL as well as MALT components. For those diagnosed with HT, we analyzed the tissues for immunoreactivity towards CD10, Bcl-6, or MUM1 according to a previous study (5). Helicobacter pylori (H. pylori) infection status was determined if either the histologic examinations or urease breath tests was positive. All the original histological slides were reviewed independently by three histopathologists.

Clinical information was obtained from the medical records. The staging procedures included routine laboratory 
tests, whole body computed tomography, endoscopy of the upper gastrointestinal tract, bone marrow aspiration, and optional positron emission tomography. The Lugano staging system, which is a modified version of the Ann Arbor criteria for gastrointestinal non-Hodgkin's lymphoma, was used for staging.

Treatment modalities and response assessment. Four therapeutic modalities were used: H. pylori eradication (HPE); chemotherapy (CT); or radiotherapy (RT); surgery (ST), or any combination. Chemotherapy included anthracycline-based or non-anthracycline regimens. The HPE regimen included proton pump inhibitors and a combination of antibiotics. Response to treatment was assessed according to the revised response criteria for malignant lymphomas (6).

Statistical analysis. Overall survival (OS) and progression-free survival (PFS) were the primary endpoints of the survival analysis. OS was considered as the time from the data of diagnosis to the date of death from any cause. PFS was determined as the time from the start of treatment to the date of treatment failure, relapse, or death from any cause. Survival curves were estimated by the Kaplan-Meier method. The life table method was used for survival estimations. Survival curves were compared using the log-rank test. Univariate analysis was performed for all variables. The Cox regression model was fitted with some variables that influenced the prognosis $(\mathrm{p}<0.05)$ in the univariate analysis to determine independent prognostic factors for survival. P-values $\leq 0.05$ were considered to be statistically significant. All reported P-values were two-tailed.

\section{Results}

Patient characteristics. Of the 71 patients with HT, 40 were male and 31 were female [male/female $(\mathrm{M} / \mathrm{F})$ ratio, 1.3:1], with a median age of 56 years (age range, 18-86 years) (Table I). Their clinical course was often insidious, lacking specific clinical presentation with the most common complaints including abdominal pain or discomfort, weight loss, poor appetite, nausea and vomiting. The majority of patients presented with good performance status (PS, 0-1 for 81\%). Routine laboratory tests, including serum LDH and $\beta 2-\mathrm{MG}$, were usually within normal limits. Macroscopically, we found that the antrum was the most commonly involved site $(48 / 71,67.6 \%)$, followed by the corpus $(39 / 71,54.9 \%)$, and fundus $(21 / 71,29.5 \%)$. According to the Lugano staging system, a larger proportion of patients had stage IE disease (45\%). Among patients who presented with stage II (35\%), 18 were diagnosed with local lymph node involvement (stage II1) and 7 had distant abdominal nodal extension (stage II2). Stages IIE and IV accounted for 12 and $8 \%$, respectively.

Survival analysis. The median time of follow-up was 52.5.9 months (range, 1-172 months). The median time to progression was 49.5 months (range, 1-138 months), and the median OS was 59.5 months (range, 2-172 months). The PFS and OS rates after 5 years were 50 and $56 \%$, respectively (Fig. 1).

Treatment outcomes. For all 71 patients, the overall response rate (ORR) was achieved at 85\%. Thirty-six (52\%), 23 (33), six (9\%), and four (6) patients attained complete remission (CR),
Table I. Characteristics of 71 patients with HT.

-

\begin{tabular}{lc} 
Characteristics & $\begin{array}{c}\text { No. of assessab } \\
\text { patients }\end{array}$ \\
\hline Sex & \\
Male & $38(53 \%)$ \\
Female & $33(47 \%)$ \\
Male/female ratio & 1.2 \\
Age (years) & \\
$\leq 60$ & $50(70 \%)$ \\
$>60$ & $21(30 \%)$ \\
Mean (range) & $55(19-81)$ \\
Lugano staging & \\
I-II & \\
IIE-IV & $56(68 \%)$ \\
Serum LDH level & $15(32 \%)$ \\
Normal & \\
Abnormal & \\
$\beta 2-M G$ & $51(72 \%)$ \\
Normal & $20(28 \%)$ \\
Abnormal & \\
m-IPI & \\
$0-1$ & $58(81 \%)$ \\
$\geq 2$ & $13(19 \%)$
\end{tabular}

Infection with H. pylori

Positive

$45(63 \%)$

Negative

$26(37 \%)$

Immunophenotype classification

GCB subtype

$30(42 \%)$

Non-GCB subtype

$41(58 \%)$

Tumor mass (cm)

$<10$

$49(69 \%)$

$\geq 10$

$22(31 \%)$

Performance status

$<2$

$58(81 \%)$

$\geq 2$

Anemia

Present

$47(66 \%)$

Absent

$24(34 \%)$

HT, histological transformation. Adverse factors for ${ }^{a} \mathrm{~m}$-IPI included age $>60$ years, serum LDH $>245$ U/l, Performance Status (PS) $\geq 2$, advanced stage lymphoma, $\geq 1$ extranodal site involvement (excluding stomach).

partial remission (PR), stable disease (SD), and progressive disease (PD), respectively.

Four therapeutic modalities (ST, CT, RT and HPE) were used in the present study. Specifically, 13 patients (18\%) were treated by ST with $(n=8)$ or without $(n=5)$ CT, 42 patients $(59 \%)$ were treated by CT with $(n=25)$ or without $(n=17)$ HPE, and 16 patients $(23 \%)$ were given RT to treat residual disease after CT with or without HPE. 
A

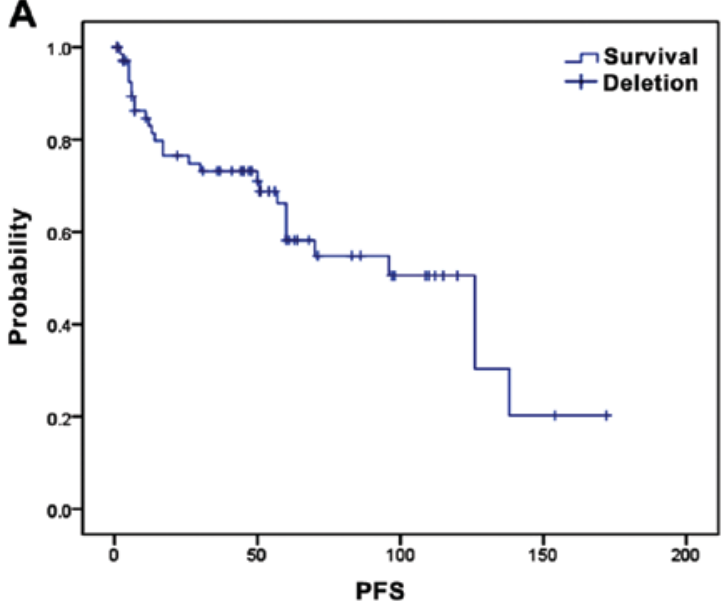

B

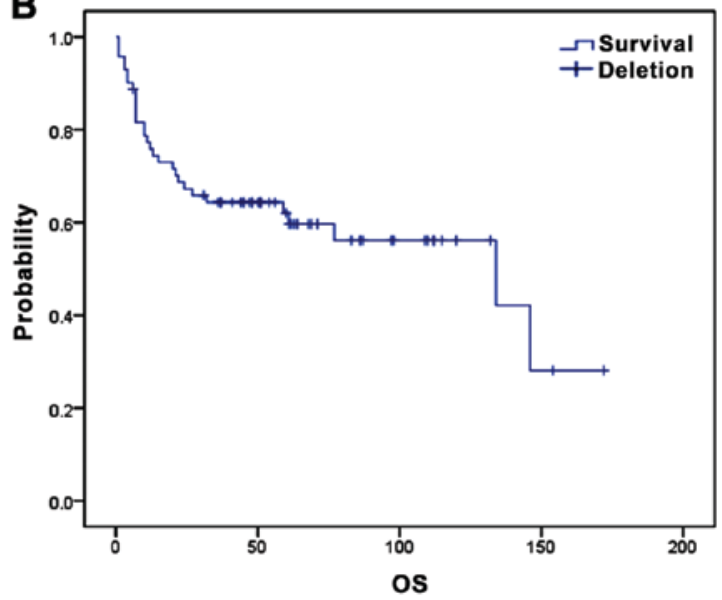

Figure 1. Survival curves for the entire population of patients with histological transformation (HT). (A) Progression-free survival (PFS) and (B) overall survival (OS).
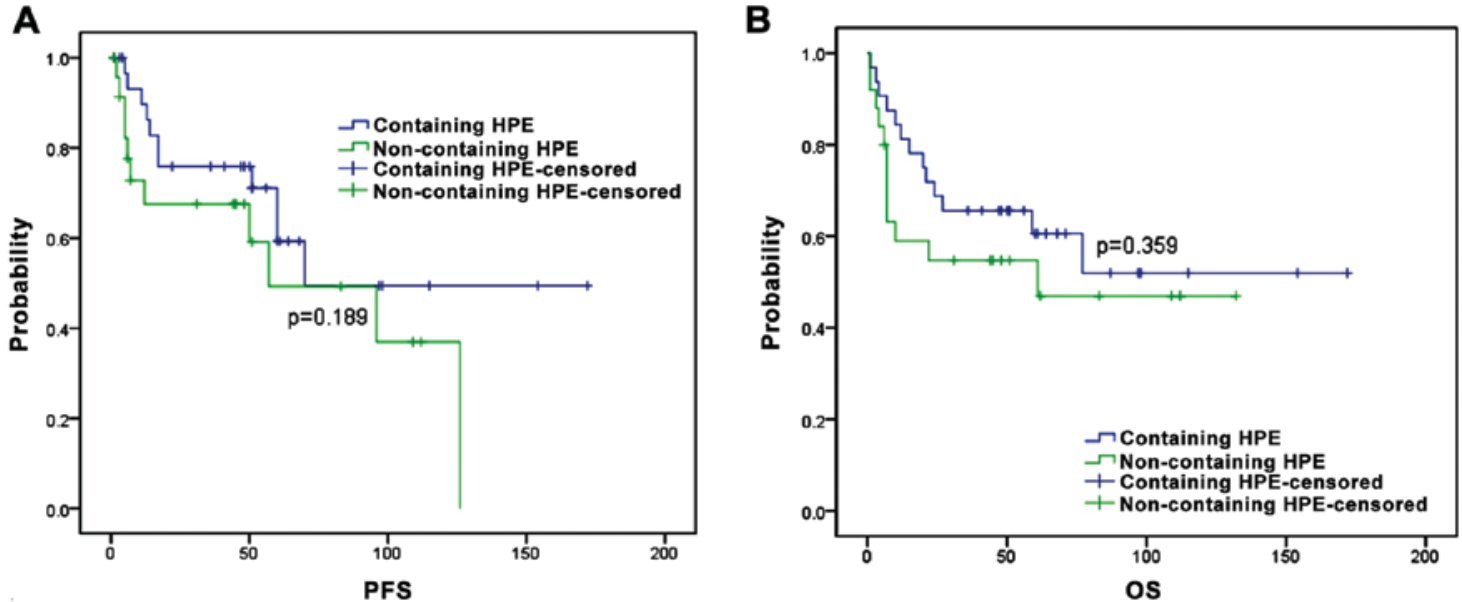

Figure 2. The progression-free survival (PFS) and overall survival (OS) of patients with histological transformation (HT) receiving therapeutic modalities containing or non-containing Helicobacter pylori (H. pylori) eradication (HPE) treatment. (A) PFS and (B) OS.

Table II. Univariate analysis of prognostic factors for PFS and OS.

\begin{tabular}{lrrrrr}
\hline & \multicolumn{2}{c}{ OS } & & \multicolumn{2}{c}{ PFS } \\
\cline { 2 - 3 } \cline { 6 - 6 } Prognostic factors & \multicolumn{1}{c}{$\chi^{2}$} & P-value & & $\chi^{2}$ & P-value \\
\hline Sex & 1.715 & 0.190 & & 2.115 & 0.146 \\
Age (years) & 2.967 & 0.085 & & 1.158 & 0.282 \\
Advanced stages & 13.516 & $<0.001$ & & 5.726 & 0.017 \\
Serum LDH $>245 \mathrm{U} / 1$ & 11.558 & 0.001 & & 7.067 & 0.008 \\
$\beta 2-M G ~>2.2 \mathrm{mg} / 1$ & 10.590 & 0.001 & & 5.050 & 0.025 \\
m-IPI $\geq 2$ & 5.931 & 0.015 & & 4.993 & 0.025 \\
Infection with H. pylori & 0.805 & 0.370 & & 1.264 & 0.261 \\
Non-GCB subtype & 1.086 & 0.257 & & 0.124 & 0.725 \\
Tumor mass $\geq 10 \mathrm{~cm}$ & 6.060 & 0.014 & & 3.949 & 0.047 \\
PS $\geq 2$ & 1.448 & 0.229 & & 4.037 & 0.045 \\
Hemoglobin $<120 \mathrm{~g} / 1$ & 0.439 & 0.507 & & 0.509 & 0.476 \\
\hline
\end{tabular}

PFS, progression-free survival; OS, overall survival; PS, performance status.
HPE was recommended as standard treatment for gMALT, while the role of H. pylori in HT has been controversial (7-9). Three patients in our study were treated with HPE as initial therapy. H. pylori was eradicated in all of them. Histological PR was achieved in 2 of them after HPE, but each experienced local relapse and then received other treatments. Among patients receiving no-surgical treatment, five-year PFS and OS estimates were compared between patients receiving HPE treatment and those receiving non-HPE treatment. We found that the 5-year PFS estimates were similar for patients receiving HPE treatment (59\%) and for those receiving non-HPE treatment $(49 \%)(\mathrm{p}=0.189)$ (Fig. 2A). The 5-year OS estimates were similar for patients receiving HPE treatment $(61 \%)$ and for those receiving non-HPE treatment $(55 \%)(p=0.359$, Fig. $2 B)$.

The influence of various prognostic factors was examined by univariate analysis. Among these, advanced stages, serum LDH, $\beta 2-\mathrm{MG}, \mathrm{m}-\mathrm{IPI}$, and tumor mass were found to adversely affected PFS and OS, while PS adversely affected PFS (Table II). Upon the Cox regression model, advanced stages were the only independent prognostic factors associated with shorter PFS, and m-IPI retained the prognostic significance for shorter PFS and OS (Table III). 
Table III. Factors retaining prognostic significance for PFS and OS with multivariate and Cox proportional hazards analysis.

\begin{tabular}{|c|c|c|c|c|c|c|}
\hline \multirow[b]{2}{*}{ Prognostic factors } & \multicolumn{3}{|c|}{ OS } & \multicolumn{3}{|c|}{ PFS } \\
\hline & $\mathrm{RR}$ & $95 \% \mathrm{CI}$ & P-value & $\mathrm{RR}$ & $95 \% \mathrm{CI}$ & P-value \\
\hline $\mathrm{LDH}>245 \mathrm{U} / 1$ & 2.785 & $0.550-14.093$ & 0.216 & 0.689 & $0.166-2.852$ & 0.607 \\
\hline Advanced stages & 3.055 & $0.955-9.774$ & 0.060 & 3.261 & $1.071-9.931$ & 0.038 \\
\hline Non-GCB subtype & 0.590 & $0.177-1.964$ & 0.389 & 3.133 & $0.809-12.130$ & 0.108 \\
\hline $\mathrm{m}-\mathrm{IPI} \geq 2$ & 9.138 & $2.743-30.444$ & 0.000 & 0.219 & $0.060-0.802$ & 0.022 \\
\hline $\mathrm{PS} \geq 2$ & 0.569 & $0.096-3.379$ & 0.535 & 0.864 & $0.170-4.402$ & 0.860 \\
\hline Hemoglobin $<120 \mathrm{~g} / \mathrm{l}$ & 1.905 & $0.651-5.581$ & 0.240 & 2.764 & $0.829-9.211$ & 0.098 \\
\hline$\beta 2-\mathrm{MG}>2.2 \mathrm{mg} / \mathrm{l}$ & 1.592 & $0.402-6.308$ & 0.508 & 2.923 & $0.734-11.639$ & 0.128 \\
\hline Tumor mass $\geq 10 \mathrm{~cm}$ & 0.579 & $0.117-2.872$ & 0.503 & 0.624 & $0.149-2.610$ & 0.519 \\
\hline
\end{tabular}

PFS, progression-free survival; OS, overall survival; PS, performance status.

\section{Discussion}

gDLBCL is the most common form of gastrointestinal lymphoma $(10,11)$. The pathogenesis of gDLBCL may have two distinct pathways (4): some gDLBCL may arise from indolent gMALT via the step of $H$.pylori-dependent HT, and the residual gDLBCLs may develop along antigen-independent pathways as de novo gDLBCL. Information and data concerning patients with HT are very limited. In our study, there was no specific clinical presentation for them with the most common complaints such as abdominal pain or discomfort, weight loss, poor appetite, nausea and vomiting. Their clinical course was indolent, duration of symptoms before diagnosis often ranged from a few weeks to several years. H. pylori infections occurred more frequently in patients with HT, compared with de novo gDLBCL in our previous study (12). It seems reasonable as HT is generally considered to arise from $H$. pylori-dependent gMALT lymphoma (13). Moreover, literature indicates HPE is effective in treating gDLBCL $(14,15)$. Our data suggested that HPE treatment tend to improve the prognosis of patients with HT, although there was no significant difference in five-year PFS/OS between the patients treated with HPE treatment and non-HPE treatment. Our results seem to further strengthen the current hypothesis (4) that the pathogenesis of gDLBCL has two distinct pathways.

Currently, there is still controversy regarding the most effective treatment strategy for gastric lymphoma $(7,8,16,17)$. Most cases of gMALT lymphoma are cured with HPE, while intensive $\mathrm{CT}$ is the mainstay of treatment for gDLBCL. As a transitional phase from gMALT lymphoma to gDLBCL, major guidelines (18) recommend the use of intensive CT with rituximab as the initial therapy for patients with HT. Nevertheless, recent studies $(16,17)$ have demonstrated the efficacy of eradication therapy for patients with HT. Morgner et al (19) reported that HPE achieved CR in six of eight patients with HT. A Taiwanese prospective study (14) also demonstrated that HPE led to CR in 10 of 16 patients with HT and none experienced recurrence during a long-term follow-up. In our study, among three patients treated with HPE as first-line therapy, histological PR was achieved in 2 of them after antibiotics. Moreover, patients receiving HPE treatment tend to have a good prognosis, although there were no statistically different on five-year PFS/OS between patients receiving HPE treatment and those receiving non-HPE treatment (49\%). This finding is consistent with recent studies. Besides, HPE is far less toxic and cheaper than chemotherapy, thus it may be a promising therapeutic approach for patients with HT. However, in order to obtain accurate benefits of HPE for patients with HT, conducting a large-scale prospective randomized clinical trial is ideal.

Prognostic factors for patients with HT have not been reported previously. In the present study, m-IPI retained their prognostic significance for shorter PFS and OS upon the Cox regression model. Previous studies $(20,21)$ have indicated that m-IPI, in gDLBCL, is an effective prognostic factor, although it has not been reported that m-IPI at HT were adverse factors for PFS/OS. In a previous study, patients in the low-risk group (m-IPI $\leq 1)$ had significantly longer survival than intermediate/high-risk (m-IPI $\geq 2$ ) patients. In our study, advanced stages were demonstrated to be an independent prognostic factor associated with shorter PFS. Findings have also shown that the difference in stages may have been associated with the difference in prognosis for gDLBCL $(22,23)$. Medina-Franco et al (23) showed that patients with early stages had a significantly better survival than patients with advanced stages. As HT and gDLBCL share many of the same biological characteristics, we believe that m-IPI or advanced stages were effective predictors for prognosis of HT.

\section{References}

1. Tomonaga M: Outline and direction of revised WHO classification of tumors of haematopoietic and lymphoid tissues. Rinsho Ketsueki 50: 1401-1406, 2009 (In Japanese).

2. Nakamura S, Ye H, Bacon CM, Goatly A, Liu H, Kerr L, Banham AH, Streubel B, Yao T, Tsuneyoshi M, et al: Translocations involving the immunoglobulin heavy chain gene locus predict better survival in gastric diffuse large B-cell lymphoma. Clin Cancer Res 14: 3002-3010, 2008.

3. Yoshino T, Omonishi K, Kobayashi K, Mannami T, Okada H, Mizuno M, Yamadori I,Kondo Eand Akagi T: Clinicopathological features of gastric mucosa associated lymphoid tissue (MALT) lymphomas: high grade transformation and comparison with diffuse large B cell lymphomas without MALT lymphoma features. J Clin Pathol 53: 187-190, 2000. 
4. Kaneko Y, Sakurai S, Hironaka M, Sato S, Oguni S, Sakuma Y, Sato K, Sugano K and Saito K: Distinct methylated profiles in Helicobacter pylori dependent and independent gastric MALT lymphomas. Gut 52: 641-646, 2003.

5. Hans CP, Weisenburger DD, Greiner TC, Gascoyne RD, Delabie J, Ott G, Müller-Hermelink HK, Campo E, Braziel RM, Jaffe ES, et al: Confirmation of the molecular classification of diffuse large B-cell lymphoma by immunohistochemistry using a tissue microarray. Blood 103: 275-282, 2004.

6. Cheson BD, Horning SJ, Coiffier B, Shipp MA, Fisher RI, Connors JM, Lister TA, Vose J, Grillo-López A, Hagenbeek A, et al; NCI Sponsored International Working Group: Report of an international workshop to standardize response criteria for non-Hodgkin's lymphomas. J Clin Oncol 17: 1244, 1999.

7. Kuo SH, Yeh KH, Wu MS, Lin CW, Hsu PN, Wang HP, Chen LT and Cheng AL: Helicobacter pylori eradication therapy is effective in the treatment of early-stage $H$ pylori-positive gastric diffuse large B-cell lymphomas. Blood 119: 4838-4844, 2012.

8. Ferreri AJ, Govi S, Raderer M, Mulè A, Andriani A, Caracciolo D, Devizzi L, Ilariucci F, Luminari S, Viale E, et al: Helicobacter pylori eradication as exclusive treatment for limited-stage gastric diffuse large B-cell lymphoma: results of a multicenter phase 2 trial. Blood 120: 3858-3860, 2012.

9. Hussell T, Isaacson PG, Crabtree JE and Spencer J: The response of cells from low-grade B-cell gastric lymphomas of mucosa-associated lymphoid tissue to Helicobacter pylori. Lancet 342: 571-574, 1993.

10. Ghimire P, Wu GY and Zhu L: Primary gastrointestinal lymphoma. World J Gastroenterol 17: 697-707, 2011.

11. Arora N, Manipadam MT, Pulimood A, Ramakrishna BS, Chacko A, Kurian SS and Nair S: Gastrointestinal lymphomas: pattern of distribution and histological subtypes: 10 years experience in a tertiary centre in South India. Indian J Pathol Microbiol 54: 712-719, 2011.

12. Li X, Xia B, Guo S, Zhan Z, Zhang L, Zhao D, Wu X and Zhang Y: A retrospective analysis of primary gastric diffuse large B-cell lymphoma with or without concomitant mucosa-associated lymphoid tissue (MALT) lymphoma components. Ann Hematol 92: 807-815, 2013

13. Hiyama T, Haruma K, Kitadai Y, Ito M, Masuda H, Miyamoto M, Tanaka S, Yoshihara M, Sumii K, Shimamoto F, et al: Helicobacter pylori eradication therapy for high-grade mucosaassociated lymphoid tissue lymphomas of the stomach with analysis of p53 and $\mathrm{K}$-ras alteration and microsatellite instability. Int J Oncol 18: 1207-1212, 2001.
14. Chen LT, Lin JT, Shyu RY, Jan CM, Chen CL, Chiang IP, Liu SM, Su IJ and Cheng AL: Prospective study of Helicobacter pylori eradication therapy in stage $\mathrm{I}(\mathrm{E})$ high-grade mucosa-associated lymphoid tissue lymphoma of the stomach. J Clin Oncol 19: 4245-4251, 2001.

15. Nakamura S, Matsumoto T, Suekane H, Takeshita M, Hizawa K, Kawasaki M, Yao T, Tsuneyoshi M, Iida M and Fujishima M: Predictive value of endoscopic ultrasonography for regression of gastric low grade and high grade MALT lymphomas after eradication of Helicobacter pylori. Gut 48: 454-460, 2001.

16. Wotherspoon AC, Doglioni C, Diss TC, Pan L, Moschini A, de Boni M and Isaacson PG: Regression of primary low-grade $\mathrm{B}$-cell gastric lymphoma of mucosa-associated lymphoid tissue type after eradication of Helicobacter pylori. Lancet 342: 575-577, 1993.

17. Zucca E and Dreyling M; ESMO Guidelines Working Group: Gastric marginal zone lymphoma of MALT type: ESMO Clinical Practice Guidelines for diagnosis, treatment and follow-up. Ann Oncol 21 (Suppl 5): v175-v176, 2010.

18. Shannon EM, MacQueen IT, Miller JM and Maggard-Gibbons M: Management of primary gastrointestinal non-Hodgkin lymphomas: a population-based survival analysis. J Gastrointest Surg 20: 1141-1149, 2016.

19. Morgner A, Miehlke S, Fischbach W, Schmitt W, MüllerHermelink H, Greiner A, Thiede C, Schetelig J, Neubauer A, Stolte M, et al: Complete remission of primary high-grade B-cell gastric lymphoma after cure of Helicobacter pylori infection. J Clin Oncol 19: 2041-2048, 2001

20. Ibrahim EM, Ezzat AA, Raja MA, Rahal MM, Ajarim DS, Mann B, Baloush A, Stuart RK and Bazarbashi SN: Primary gastric non-Hodgkin's lymphoma: clinical features, management, and prognosis of 185 patients with diffuse large B-cell lymphoma. Ann Oncol 10: 1441-1449, 1999.

21. Cortelazzo S, Rossi A, Roggero F, Oldani E, Zucca E, Tondini C, Ambrosetti A, Pasini F, Pinotti G, Bertini M, et al; International Extranodal Lymphoma Study Group (IELSG): Stage-modified international prognostic index effectively predicts clinical outcome of localized primary gastric diffuse large B-cell lymphoma. Ann Oncol 10: 1433-1440, 1999.

22. Psyrri A, Papageorgiou S and Economopoulos T: Primary extranodal lymphomas of stomach: Clinical presentation, diagnostic pitfalls and management. Ann Oncol 19: 1992-1999, 2008.

23. Medina-Franco H, Germes SS and Maldonado CL: Prognostic factors in primary gastric lymphoma. Ann Surg Oncol 14: 2239-2245, 2007 